\title{
Candida rugosa Candidaemia in a Critically Ill Trauma Patient Successfully Treated with Amphotericin B
}

\author{
Authors \\ Santwana Verma, Archana Angrup, Vineeta Sharma, M. R. Shivaprakash, \\ Sunita Gupta \\ ${ }^{1,3}$ Dept. of Microbiology, I.G.M.C, Shimla \\ ${ }^{2,4,5}$ Dept. of Microbiology, PGIMER, Chandigarh
}

\begin{abstract}
Candidemia is a common life threatening condition occurring in the hospitalized patients specially those admitted to the intensive care units. Candida rugosa is an uncommon species and a significant cause of candidemia acquired in intensive care unit. It is associated with trauma, total parenteral nutrition and central venous catheterization. The geographical distribution is limited and antifungal susceptibility of isolates demonstrate great variability. We report the case of a patient with trivial trauma following a fall while playing cricket who became critically ill with multi-organ involvement following Candida rugosa candidaemia. He was instituted amphotericin B intravenously in a dose of $1 \mathrm{mg} / \mathrm{kg}$ and showed favourable response in a week with recovery after 27 days of therapy.
\end{abstract}

Keywords: amphotericin B, Candida rugosa, candidaemia, central venous catheter, nosocomial.

\section{Introduction}

Candida rugosa has attained the status of emerging yeast constituting nearly $0.4 \%$ of all Candida isolates and become relevant due to its resistance to antifungal agents ${ }^{[1]}$. It is recently reported as a significant cause of intensive care unit (ICU)acquired candidemia ${ }^{[2]}$. Infection often results in devastating consequences allied with high fatality ${ }^{[3]}$. Association of Candida rugosa has been established in trauma cases, patients on total parenteral nutrition, central venous catheterization or other interventions. Geographical distribution is limited with vast differences in antifungal susceptibility of isolates from different regions thus the identification in new regions is vital. The occurrence of $\mathrm{C}$. rugosa in the sub-Himalayan region is not documented thus we present this first report of C. rugosa candidemia. This knowledge may help to divulge unexplained fatalities in critical patients and consider a revised clinical diagnosis in morbid cases.

\section{Case Report}

A 17 years old male child was admitted to our tertiary care hospital with complaints of pain both lower limbs, following a fall while playing cricket. Subsequently, the child developed tenderness of calves, thighs and restricted movement at hip joints with inability to walk. There were no complaints of abnormal body movements, numbness, parasthesias or bladder and bowel dysfunction. History was negative for birth asphyxia, tuberculosis and arthritis. 
General physical examination revealed normal vitals. Musculoskeletal examination revealed normal bulk of muscles with absence of swelling or redness. There was tenderness of bilateral calves and thighs with elevated temperature over left thigh with restricted movements at hip joints. The child progressed rapidly to septic shock with difficulty in breathing, chest pain, cough, fever and respiratory rate of 50 per minute, nasal flaring, suprasternal recession and SPO2 of $78 \%$. Crepitations were auscultated bilaterally in infrascapular, infraaxillary and inframammary regions.

Septic arthritis complicated by septic shock and respiratory distress was suspected. Magnetic resonance imaging revealed a large collection of pus between semimembranous biceps formis muscles measuring 26x4.8 cm with normal underlying femur bone. Pus was evacuated surgically and culture grew Staphylococcous aureus susceptible to linezolid, clindamycin and gentamicin and resistant to erythromycin and cotrimoxazole. Treatment was initiated with linezolid and empiric meropenem.

Post operative course was complicated by development of acute respiratory distress syndrome, severe shock and dehydration. Further management was done with mechanical ventilation, multiple transfusions of blood and fresh frozen plasma. Nasogastric tube was inserted, central venous catheter (CVC) line secured and vitals and electrolytes maintained.

At the end of one week post-operatively, patient developed bilateral crepitations in lungs, fever of $1030 \mathrm{~F}$ with progressive acute respiratory distress syndrome (ARDS), multi-organ distress syndrome (MODS) and had blood stained endotracheal secretions, bleeding from nasogastric tube and haematuria. Cultures of blood and sputum samples grew Enterobacter aerogenes sensitive to ciprofloxacin and meropenem and treatment was modified to include ciprofloxacin. On day 12 postoperatively, fever spiked to $1040 \mathrm{~F}$ despite extensive antimicrobial coverage as per susceptibility pattern. CVC line was changed and culture of removed CVC and repeat blood culture revealed pure growth of creamy, irregular pasty colonies (Figure 1) showing oval budding yeast 3-6 $\mu \mathrm{m}$ in size in microscopy. Germ tube test was negative consistent with nonalbicans Candida species. The isolate was confirmed as Candida rugosa at the National Culture Collection of Pathogenic Fungi, Post Graduate Institute of Medical Education and Research, Chandigarh (accession number 470096). Injection Amphotericin B, $1 \mathrm{mg} / \mathrm{kg}$ was started. On day 5 of therapy, child recorded normal body temperature, was put off ventilator and was able to communicate with gestures. By day 10 the child was accepting feeds orally and recovering satisfactorily. Antifungal treatment was continued for 27 days and child discharged after recovery.

\section{Discussion}

Candida rugosa has emerged as an agent of fatal candidaemia in the recent past. It was first isolated from human feces by Anderson in 1917 and named Mycoderma rugosa $^{[4]}$. The yeast has been recovered from bovine droppings, stale butter, margarine factory, seaweeds and cattle with mastitis. It is utilized as a source of lipases for commercial synthesis of esters. The first human case was reported in 1985 by Reinhart et al and since then limited reports of outbreaks from burn units and other critically ill trauma patients or individual cases are documented ${ }^{[4]-[8]}$.

The predisposing factors include administration of multiple antimicrobials, cancer chemotherapy, bowel surgery, use of intravenous plastic catheters and chronic total parenteral nutrition ${ }^{[5]}$. An in-situ CVC line has been recognized as the single most relevant threat ${ }^{[5]}$. Speculations exist as to the possible source of human infections and accounts of outbreaks or clusters of hospitalized cases establish the occurrence of $C$. rugosa candidemia in nosocomial settings. Singh et al documented 25 isolates of $\mathrm{C}$. rugosa representing $18.4 \%$ of all Candida isolated from critically ill trauma cases where random amplification of polymorphic DNA established a uniform pattern with a probable common source that remained obscure ${ }^{[5]}$. Genetic relatedness was proven in the six hospitalized $\mathrm{C}$. rugosa candidemia cases from Brazil supplementing 
nosocomial origin of these infections ${ }^{[6]}$. It has been identified on fomites in institutions and reported to be transmitted between patients ${ }^{[9]}$. Our subject developed fungemia several days after hospitalization possibly representing a nosocomial affliction.

The geographical distribution of C. rugosa is limited with most isolates from Latin America, others from Asia- Pacific or Europe and least from North America $^{[1]}$. Candidemia due to $C$. rugosa is documented in Indian literature from critically ill trauma patients from New Delhi, a comatose diabetic female from Assam, intravenous catheterrelated infection from Mangalore and in patients admitted to intensive care units [2], [5], [8], [10]

Reduced susceptibility of C.rugosa to antifungal agents is of grave concern. Resistance to nystatin was apparent in a study reporting 15 episodes of candidaemia due to C.rugosa following topical nystatin in a burn unit ${ }^{[7]}$. In vitro sensitivity reported $40.5 \%$ and $61.4 \%$ susceptibility to fluconazole and voriconazole respectively ${ }^{[1]}$. The occurrence of fatal breakthrough infection with $\mathrm{C}$. rugosa in patients on amphotericin B is in contrast to observations of favourable susceptibility reports to amphotericin B, flucytosine and voriconazole ${ }^{[5]-[7]}$. In our experience, successful management was done with amphotericin $\mathrm{B}$ in the present case.

In view of contrasting reports of antifungal susceptibilities of different isolates, each case and isolate needs to be considered individually. Voriconazole, posaconazole and echinocandins are suggested therapeutic alternatives ${ }^{[9], ~[11] . ~}$

\section{Conclusion}

Candidaemia is a life threatening infection of hospitalized patients. Most species isolated are susceptible to commonly used antifungal drugs. Candida rugosa is a rare species associated with trauma, central venous line and total parenteral nutrition. Response to various antifungal agents is unpredictable and no set guidelines are available. Thus, it is important to suspect candedaemia due to Candida rugosa in severely ill patients not responding to therapy and manage it as an emergency.

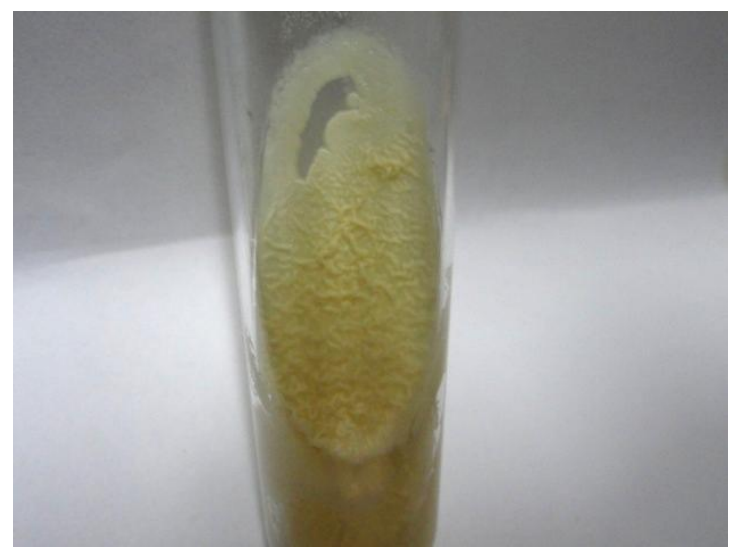

Fig. 1 Cream coloured irregular pasty colonies of Candida rugosa on SDA with chloramphenicol.

\section{References}

1. M. A. Pfaller, D.J. Diekema, A.L. Colombo, C. Kibber, K.P.Ng, D.L. Gibbs, "Candida rugosa, an emerging fungal pathogen with resistance to azoles: Geographic and temporal trends from the ARTEMIS DISK AntifungalSurveillance Program," J Clin Microbiol., vol. 44(10),pp. 3578-3582, 2006

2. Chakrabarti, P.Sood, S.M. Rudramurthy, S. Chen, H. Kaur, M. Capoor, "Incidence, characteristics and outcome of ICU-acquired candidemia in India," Intensive Care Medicine, vol. 41(2), pp. 285-295, 2015.

3. Behera, R.I. Singh, I. Xess, P. Mathur, F.Hasan, M. C. Misra, "Candida rugosa: a possible emerging cause of candidaemia in trauma patients," Infection, vol. 38(5), pp.387-393, 2010.

4. J.F. Reinhardt, P. J. Ruane, L. J. Walker, W. L. George, "Intravenous catheter-associated fungemia due to Candida rugosa," J Clin Microbiol., vol. 22(6), pp. 1056-1057, 1985.

5. R. I. Singh, I. Xess, P. Mathur, B. Behera, B. Gupta, M. C. Misra, "Epidemiology of candidaemia in critically ill trauma patients; experience of a level I trauma centre in North India," J Medical Microbiol., vol. 60, pp. 342-348, 2011.

6. L. Colombo, A. S. Melo, R. F. Crespo Rosas, R. Salomao, M. Briones, R. J. Hollis, "Outbreak of Candida rugosa candidaemia: an emerging pathogen that may be refactory 
to amphotericin B therapy," Diagn Microbiol Infect Dis., vol. 46(4), pp. 253-257, 2003.

7. M. P. Dube, P. N. Heseltine, M. G. Rinaldi, S. Evans, B. Zawacki, "Fungemia and colonization with nystatin - resistant Candida rugosa in a burn unit," Clin Infect Dis., vol. 18, pp. 77-82, 1994.

8. B. S. Dutta, A. Sharma, N. K. Hazarika, P. Barua, "Candida rugosa - An emerging cause of nosocomial candidaemia," Indian Journal of Public Health Research and Development, vol. 4(3), pp. 49-52, 2013.

9. L. R. Minces, K. S. Ho, P. J. Veldkamp, C. J. Clancy, "Candida rugosa: a distinctive emerging cause of candidaemia. A case report and review of the literature," Scand J Infect Dis., vol. 41, pp. 892-897, 2009.

10. S. Shenoy, M. Samuga M, S. Urs, K.M. Anuradha, M. M. Kurian, A. Augustine, "Intravenous catheter- related Candida rugosa fungaemia," Trop Doct. vol. 26(1), 31, 1996.

11. S. Hernandez, G. M. Gonzalez, D. I. Mc Carthy, A. L. Colombo, L. K. Najvar, R. Bocanegera, J. R. Garybill, "Alternatives to amphotericin B for Candida rugosa infection," J of Antimicrobial Chemotherapy, vol. 54, pp. 477-480, 2004. 